\title{
The causality between smoking and lung cancer among groups and individuals: addressing issues in tobacco litigation in South Korea
}

\author{
Young-Ho Khang \\ Department of Health Policy and Management, Seoul National University College of Medicine, and Institute of Health Policy and Management, \\ Seoul National University Medical Research Center, Seoul, Korea
}

This article discusses issues on the causality between smoking and lung cancer, which have been raised during the tobacco litigation in South Korea. It should be recognized that the explanatory ability of risk factor(s) for inter-individual variations in disease occurrence is different from the causal contribution of the risk factor(s) to disease occurrence. The affected subjects of the tobacco litigation in South Korea are lung cancer patients with a history of cigarette smoking. Thus, the attributable fraction of the exposed rather than the population attributable fraction should be used in the tobacco litigation regarding the causal contribution of smoking to lung cancer. Scientific evidence for the causal relationship between smoking and lung cancer is based on studies of individuals and groups, studies in animals and humans, studies that are observational or experimental, studies in laboratories and communities, and studies in both underdeveloped and developed countries. The scientific evidence collected is applicable to both groups and individuals. The probability of causation, which is calculated based on the attributable fraction for the association between smoking and lung cancer, could be utilized as evidence to prove causality in individuals.

KEY WORDS: Causality, Legislation and jurisprudence, Lung cancer, Smoking, South Korea, Tobacco

\section{INTRODUCTION}

Tobacco litigation in South Korea (hereafter Korea) is ongoing. In April 2014, the National Health Insurance Service (NHIS) in Korea filed a suit for compensatory damages caused by smoking against tobacco companies such as KT\&G, Philip Morris Korea Inc., and BAT Korea Ltd.This litigation is on the damages of 53.7 billion Korean won which was paid by the NHIS to 3,484 patients who had a 20 pack-year or more history of smoking and who smoked for 30 years or more. These patients developed

Correspondence: Young-Ho Khang

Department of Health Policy and Management, Seoul National University

College of Medicine, 103 Daehak-ro, Jongno-gu, Seoul 110-799, Korea

Tel: +82-2-740-8361, Fax: +82-2-743-2009, E-mail: yhkhang@snu.ac.kr

Received: Apr 21, 2015, Accepted: May 31, 2015, Published: May 31, 2015

This article is available from: http://e-epih.org/

(C) 2015, Korean Society of Epidemiology

(C) This is an open-access article distributed under the terms of the Creative Commons Attribution License (http://creativecommons.org/licenses/by/3.0/), which permits unrestricted use, distribution, and reproduction in any medium, provided the original work is properly cited. one of three major cancers, which had the great magnitude of causal relationship with smoking, including small cell lung cancer, squamous cell lung cancer, and squamous cell laryngeal cancer. The NHIS explained that the litigation was limited to these cancer types this time, for which the causal relationship between smoking and disease occurrence was recognized by previous tobacco litigation [1]. A trial regarding the causality between smoking and lung cancer was held at the Seoul Central District Court in January 2015. Issues on the causality will be dealt in ongoing trials.

In terms of the causality between smoking and lung cancer, tobacco companies use the following logic [2]. First, "because the evidence for the causality between smoking and lung cancer is merely a statistical association derived from epidemiologic studies in population, it cannot provide information about the cause(s) of disease in individual lung cancer patients' [2]. Second, 'long-term smoking does not cause lung cancer in all smokers, and not all lung cancer patients are smokers. The occurrence of lung cancer is also associated with indoor and outdoor air pollution, occupational exposure to hazardous substanc- 
es, alcohol drinking, and dietary habits. Therefore, it is necessary to prove with a very high likelihood that a certain patient would not have had lung cancer if he/she had not smoked by investigating the smoking history, progression of disease occurrence, medical records, and other information of each individual for the 3,484 people whose medical bills were paid by the NHIS' [2].

There are several issues related to tobacco litigation. In addition to causality, the illegal conduct of tobacco companies is a very important legal issue. However, in this article, the discussion is limited to causality from an epidemiological perspective. While observing the progress of tobacco litigation in Korea, the author identified three discussion topics regarding the causality between smoking and lung cancer. First, there was confusion about the explanatory ability of risk factor(s) for inter-individual variations in disease occurrence and the causal contribution of risk factor(s) to disease occurrence. Second, there were issues with the applicability of evidence for the causality between smoking and lung cancer to population. Third, the applicability of the evidence for the causality between smoking and lung cancer at the level of individuals should be discussed.

\section{INTER-INDIVIDUALVARIATIONS IN DISEASE OCCURRENCEAND CAUSAL CONTRIBUTION}

The explanatory ability of a specific risk factor for inter-individual variations in disease occurrence and the magnitude of causal contribution of a specific risk factor to disease occurrence are two very different issues [3]. Despite the difference, these two issues appear in a debate on the causes of disease. For example, the recent debate associated with a paper published in the journal Science in early January 2015, which addressed the role of chance in cancer occurrence, has originated because of confusion regarding two issues: variations at the tissue level and causal contribution $[4,5]$.

Regarding the causality of smoking and lung cancer, inter-individual variations in disease occurrence can be summarized as 'there are non-smokers among lung cancer patients, and about $10 \%$ of all smokers develop lung cancer' [2]. This is one basis of argument by tobacco companies against the causal association between smoking and lung cancer at an individual level [2]. How can inter-individual variations (i.e., some people develop lung cancer but others do not) be explained? How much is the ability of smoking in explaining inter-individual variation of lung cancer? Does the level of this explanatory ability refer to the magnitude of the causal contribution?

Inter-individual variations can be explained by heritable factors and both shared and non-shared environmental factors [3,6]. The sum of all proportions for these three factors explaining the inter-individual variations in disease occurrence is $100 \%$.
This point is distinct from the indicators used to evaluate the causal contribution of specific factors to disease occurrence, i.e., attributable fraction and population attributable fraction. If the attributable fraction of different risk factors is added, it should theoretically be $>100 \%[7,8]$.

When heritable factors explain most of the inter-individual variations, the disorder is considered genetic. However, environmental factors can also contribute to genetic diseases. Phenylketonuria (PKU) is a congenital metabolic disorder that is caused by the $P K U$ gene and a phenylalanine diet. However, PKU is considered a genetic disorder because few people have the gene, whereas most people have a phenylalanine (an amino acid common in most animal and plant proteins) diet. If all individuals in a certain population group have PKU but only some have a phenylalanine diet, PKU would be seen as an environmental disease (caused by diet). In fact, current treatment of PKU involves a phenyalanine-free diet, which is a totally environmental intervention.

Shared environmental factors are those that would be experienced by twins, such as passive smoking and identical dietary habits in a household during childhood [6]. The degree to which environmental factors not shared by twins explain inter-individual variations in disease occurrence is the explanatory ability of non-shared environmental factors. Cigarette smoking beginning after adolescence is included. For most health problems, the explanatory ability of non-shared environmental factors is the greatest [9]. Among non-shared environmental factors, the most significant factor is considered chance, a stochastic factor $[3,9]$. According to a previous study examining the degree to which heritable, shared environmental, and non-shared environmental factors explain inter-individual variations in cancer occurrence, the degree that non-shared environmental factors explain inter-individual variations was the greatest [6]. For lung cancer, the extent to which heritable, shared environmental, and nonshared environmental factors explained inter-individual variations in lung cancer occurrence was $26 \%, 12 \%$, and $62 \%$, respectively [6].The ability of smoking to explain inter-individual variations in lung cancer would be approximately $10 \%$ [10], which would be relatively high compared to other risk factors.

Heritability, the extent to which heritable factors explain inter-individual phenotypic variations, does not describe the magnitude of causal influence of heritable factors on disease occurrence [11]. Even if the ability of a specific factor in explaining inter-individual variations is not large, it does not mean that the causal influence of the pertinent factor is small. Even if the explanatory ability of smoking for inter-individual variations is small, it does not change the fact that smoking is a strong cause of lung cancer. For example, if all individuals in a certain society have smoked one pack of cigarettes per day for 20 years, smoking could not explain inter-individual variations in lung 
cancer occurrence. This is because all individuals smoked identically. In this society, lung cancer could be considered a genetic disorder or a disease derived by a chance [3,12]. However, the incidence of lung cancer in the societal level would be very high, because smoking causally contributes to lung cancer.

The causal contribution of a specific factor to disease occurrence is expressed as the attributable fraction. The attributable fraction among the exposed and the population attributable fraction are utilized. For example, results of domestic research on the population attributable fraction of smoking for lung cancer [13] has been cited by a tobacco company in the tobacco litigation in Korea [2]. However, the attributable fraction of the exposed should be used in tobacco litigation, because the affected individuals of the litigation are lung cancer patients with a smoking history.

\section{MULTI-CAUSAL MODEL OF DISEASE OCCURRENCE AND SMOKING}

In the tobacco litigation in Korea, lung cancer is not called a 'specific disease' in which the cause unequivocally corresponds to the effect [2,14]. Instead, it is a 'non-specific disease' in which several causal factors play a role in occurrence $[2,14]$. In terms of the relationship between smoking and lung cancer, tobacco companies argue that the relationship is not 1:1 (i.e., without A, $B$ does not exist). However, a 1:1 correlation of cause and effect as well as a mono-cause $\mathrm{A}$ to meet the necessary and sufficient conditions for the occurrence of disease B cannot exist [15]. The categorization of a disease itself as specific and non-specific cannot be accepted in epidemiology. This categorization is based on the Korean Supreme Court verdict regarding the litigation on Agent Orange exposures in Vietnam War. However, the inappropriateness of this categorization has been addressed in a domestic study [16].

Infectious diseases could be considered specific disease based on the above definition, but they are not. Many Koreans have Mycobacterium tuberculosis (MTB) in their body but there are few tuberculosis (TB) patients in the entire population [17]. Under specific conditions such as nutritional deficiency, hygiene issues, and immune function abnormalities, TB can develop. Of all the individuals infected with Vibrio cholerae, a few actually display classic cholera symptoms including severe watery diarrhea [18]. Vibrio cholerae and MTB are 'necessary conditions' in the occurrence of TB and cholera, respectively. However, with the exception of infectious diseases, most non-communicable diseases do not have necessary conditions. Risk factors for most non-communicable diseases are component causes [19]. The attributable fraction of Vibrio cholerae in cholera and MTB in TB is $100 \%$. If an individual is not infected with Vibrio cholerae, he/she will not contract cholera. Likewise, if one is not infected with MTB, he/she will not contractTB. If specificity refers to the magnitude of causal contribution of risk factors (Vibrio cholerae and $\mathrm{MTB}$ ) to diseases (cholera and TB, respectively), then smoking, for which the attributable fraction for lung cancer is $90 \%$, is a specific factor compared to the other various risk factors. The level of specificity is even stronger for small cell lung cancer, squamous cell lung cancer, and squamous cell laryngeal cancer at issue in current tobacco litigation. If the disease classification system changes and 'smoking-induced lung cancer', which consists of a considerable proportion of the entire lung cancers, becomes a separate disease category, then smoking would become a necessary condition and the level of specificity would be greater.

Several factors affect disease occurrence. In principle, no disease develops from one cause. However, the fact that several causes are involved in disease occurrence does not counteract the causal effect of smoking on lung cancer. Smoking is a very strong cause of lung cancer, and the causal effect of smoking on lung cancer is 'specific' and greater than other known specific risk factors. This is especially true for three types of cancer (small cell lung, squamous cell lung, and squamous cell laryngeal cancers), which are at issue in current tobacco litigation.

\section{WHERE DOESTHE EVIDENCE REGARDING CAUSALITY BETWEEN SMOKING AND LUNG CANCER ORIGINATE?}

Based on observations of current tobacco litigation, it appears that tobacco companies are creating a deep and wide gap between groups and individuals regarding the applicability of evidence on causality between smoking and lung cancer. For example, the following arguments have been made by tobacco companies [2]. 'Because the evidence for the causality between smoking and lung cancer is derived from epidemiologic studies in population, it is not possible to apply the pertinent study results to individuals' [2]. 'Even if smoking contributes to $80 \%$ $90 \%$ of lung cancer occurrence, the contribution cannot be applied to individuals because this is a statistical result observed in population group(s) and the role of other factors in individuals cannot be ignored' [2]. 'Epidemiological study results about the association between smoking and lung cancer imply merely general causation but do not provide information about specific causation in individuals' [2].

Epidemiological studies have played multiple roles in verifying the causality between smoking and lung cancer and quantifying the extent of harmfulness of smoking. However, in establishing the evidence for the causality between smoking and lung cancer, other observational and experimental results (i.e., indi- 
vidual observations, animal studies, and laboratory chemical analyses) have also contributed significantly [20].

First, there are the results of animal experiments. These include studies that demonstrated cancer development when 'tobacco juice' was administered to animals. Wynder and colleagues induced carcinogenesis by applying cigarette tar to mice [21].

Second, there are observational studies of cellular pathology. Since the 1930s, pathologists determined that smoking impairs the movement of cilia (a cellular organelle in the human body that sweeps foreign materials out of the lungs) in the upper airway causing ciliostasis [20]. Cellular pathologic studies have found that upon ciliostasis, the cigarette contents inhaled become trapped in the lungs, which can cause cancer.

Third, carcinogenic chemicals have been found in cigarette smoke. In the 1930s, Angel Roffo identified polycyclic aromatic hydrocarbons present in cigarette smoke [20], and in 1952 researchers in the tobacco company Brown and Williamson, identified benzpyrene [22]. Afterwards, numerous carcinogens were identified through chemical analysis. Indeed, approximately 7,000 chemicals are produced during smoking, which may have harmful effects on human [23].

In evaluating causality, epidemiologists also use data from animal experiments, pathologic observations of individuals, and laboratory chemical analyses. In the field of epidemiology, Hill's considerations for causation have been utilized [24]. Considerations such as biological plausibility, coherence, experiments, and analogies require the results of animal experiments, laboratory results, and observational results from individuals. Activities that describe epidemiological study results merely as 'statistical association' and both disparage and restrict the role(s) of epidemiology in determining causality between smoking and lung cancer in individuals can be seen as attempts to prevent the epidemiological results on causality between smoking and lung cancer from being accepted in the court as the evidence for the causality. In a domestic paper in Korea, the argument that epidemiological study results cannot be used as an evidence for causality in individuals was regarded to be unreasonable [25]. Scientific evidence for the causality between smoking and lung cancer is based on data from individuals and groups, studies in animals and humans, observational and experimental studies, studies in laboratories and communities, and studies in both underdeveloped and developed countries. Therefore, the denial of the causality between smoking and lung cancer cannot help but be considered denial of the current scientific knowledge system.

Evidence for disease causation is based on study results that have been observed or verified through studies of individuals and groups, and the results applied to groups and individuals. The goal of health policies that can decrease smoking rates is to reduce the incidence and mortality from diseases such as lung cancer in population. The reason that smoking cessation is recommended and that treatments are provided to smokers in smoking cessation clinics is to reduce the possibility that at risk smokers develop a disease including lung cancer. Smoking cessation is recommended to smokers in a household by family members because of concerns about adverse health effects. All are based on the recognition of disease causation (at both the individual and group levels) between smoking and lung cancer. Practically, it would not be possible to say to family members and physicians who recommend smoking cessation to individual smokers, 'You are irrationally applying data observed in population to individuals'. A deep and wide gap that cannot be filled is not present between individuals and groups.

\section{CAUSALITY BETWEEN SMOKING AND LUNG CANCER IN GROUPS AND INDIVIDUALS}

The causal contribution of a specific risk factor to disease occurrence in population is expressed as the attributable fraction. If lung cancer patients with history of smoking as a group argue about the cause under conditions, then it is reasonable to conclude that smoking is responsible for lung cancer in the group. For example, if it is assumed that the relative risk of smoking for small cell lung, squamous cell lung, and squamous cell laryngeal cancers is 10 -fold higher, that is, the attributable fraction of the exposed is $90 \%$, then it can be concluded that $90 \%$ of the lung cancer incidence in the group is attributable to smoking.

An issue arises in cases in which the cause of the disease is argued at the individual level. The issue is whether the attributable fraction derived from population data is applicable to individuals. In this case, the attributable fraction, an indicator of population group(s), can be used to estimate the probability of causation (PC).

According to a dictionary of epidemiology by Porta [24], for a given case, the probability that exposure played a role in disease occurrence is termed PC. Unlike attributable fraction, which uses incidence data of population group(s), the PC addresses the probability that a randomly selected individual develops a disease that is caused by a specific exposure. This is the probability at the individual level, which is used to determine rules or legal standards [26,27].

There is a particular relationship between attributable fraction and PC. The PC is equal to or greater than the attributable fraction $[15,19,24]$. We considered an example in the dictionary of epidemiology by Porta [24]. It was assumed that 100 individuals all survived for eight years due to a wrong treatment. However, if these individuals had not received the wrong treatment, all would have survived for 10 years. In this case, because 100 survived for less than two years due to receiving the wrong 
reatment, the PC of the incorrect treatment for the shortened survival period was $100 \%$. However, the attributable fraction was calculated using person-time data, which considered the follow-up period. If the individuals were followed for 10 years, the mortality rate of the individuals who received the wrong treatment would be $100 / 800$ person-years $(0.125 / \mathrm{yr})$. If they did not receive the treatment, the mortality rate would be $100 /$ 1,000 person-years $(0.100 / y r)$. In this case, the attributable fraction is $(0.125-0.100) / 0.125=20 \%$. This is very low compared to the $100 \%$ PC [24].

Disease occurrence can be assumed to occur at a constant rate over time. If all 100 individuals in the above example who received the incorrect treatment died during follow-up, the PC would be $100 \%$. If death occurred at a constant interval during follow-up period, the sum of the follow-up period of individuals who received the wrong treatment would be approximately 500 person-years. Because the mortality rate would be 100/1,000 person-years for individuals if they did not receive the treatment, and the mortality rate of people who have received the treatment was 100/500 person-years, the attributable fraction would be $50 \%$ and the mortality rate would be two-fold higher than that of non-exposed individuals. This implies that if the attributable fraction is $50 \%$ when the disease occurs at a constant interval (closer to a real case), the PC would be approximately $100 \%$. If the attributable fraction of a certain risk factor to a disease was $90 \%$, what would the PC be? The probability that the pertinent risk factor played a role in disease occurrence in a randomly selected patient of the disease would be at least $90 \%$. A detailed study to determine the cases of lung cancer that are legally attributable to smoking would be necessary, similar to that performed during tobacco litigation in Quebec, Canada [28]. In summary, the argument that the attributable fraction derived from population can provide no information regarding causality among individuals cannot be accepted. The attributable fraction information regarding the relationship between smoking and lung cancer can be utilized to prove causality in both groups and individuals [15].

\section{CONCLUSION}

Debate on the causality between smoking and lung cancer exists in academic fields and in court. However, the views of lung cancer patients have not been clearly expressed. Lung cancer is a frightening disease. Patients have trouble breathing prior to death. Numerous patients have suffered and died in Korea, and currently many people live with the agony of lung cancer. Their suffering is not fate or their responsibility because the cause(s) is external. Tobacco litigation is one means of discovering the cause(s), which is specific to patients. Litigation can there- fore be used to represent the weak.

Smoking is the main culprit of social inequality in health. The mortality rate of lung cancer is high among individuals with low education levels or incomes [29,30]. The smoking rate is high in lower social classes [31]. In Korea, smoking plays a significant role in socioeconomic inequalities in mortality rate [32]. Smoking threatens the right to life of an individual because of the possibility of developing many diseases including lung cancer. In addition, it also creates inequality in health by increasing morbidity and mortality, especially in the socioeconomically vulnerable groups. Therefore, regulating smoking is an act of social justice.

According to prior studies, regulations on unhealthy commodities such as tobacco, alcohol, ultra-processed food, and beverages have shown a particular convergence trend [33]. That is, the better the regulation of tobacco, the better the regulation of alcohol and food. According to the Global Burden of Disease Study, the disease burden caused by these harmful goods is very large in Korea [34]. If the negative effects of the harmful goods on health were highlighted through ongoing tobacco litigation, this would be a historical footstep towards a society in which all individuals can live healthy lives in Korea.

\section{ACKNOWLEDGEMENTS}

This article is based on the author's paper presented at the international and domestic experts invited seminar, 'The causality between smoking and lung cancer, the meaning of epidemiological evidence' held by the National Health Insurance Service on April 6, 2015.

\section{CONFLICT OF INTEREST}

The author has no conflicts of interest to declare for this study.

\section{SUPPLEMENTARY MATERIAL}

Supplementary material is available at http://www.e-epih.org/.

\section{REFERENCES}

1. Kim YJ. Health Insurance Service files suit against tobacco companies [cited 2015 Mar 23]. Available from: http://www.hani.co.kr/arti/ english_edition/e_national/632850.html.

2. Preparatory pleadings for KT\&G, Phillip Morris International Korea, British American Tobacco Korea BAT in the compensation claim 2014GaHap525054 (Korean). 
3. Smith GD. Epidemiology, epigenetics and the 'Gloomy Prospect': embracing randomness in population health research and practice. Int J Epidemiol 2011;40:537-562.

4. Tomasetti C, Vogelstein B. Cancer etiology. Variation in cancer risk among tissues can be explained by the number of stem cell divisions. Science 2015;347:78-81.

5. BBC World Service. Bad luck and cancer [cited 2015 Mar 23]. Available from: http://www.bbc.co.uk/programmes/p02g0cmh.

6. Lichtenstein P, Holm NV, Verkasalo PK, Iliadou A, Kaprio J, Koskenvuo M, et al. Environmental and heritable factors in the causation of cancer--analyses of cohorts of twins from Sweden, Denmark, and Finland. N Engl J Med 2000;343:78-85.

7. Rockhill B, Newman B, Weinberg C. Use and misuse of population attributable fractions. Am J Public Health 1998;88:15-19.

8. Rowe AK, Powell KE, Flanders WD. Why population attributable fractions can sum to more than one. Am J Prev Med 2004;26:243249.

9. Plomin R. Commentary: why are children in the same family so different? Non-shared environment three decades later. Int J Epidemiol 2011;40:582-592.

10. Pearce N. Epidemiology in a changing world: variation, causation and ubiquitous risk factors. Int J Epidemiol 2011;40:503-512.

11. Burton PR, Tobin MD, Hopper JL. Key concepts in genetic epidemiology. Lancet 2005;366:941-951.

12. Rose G. Sick individuals and sick populations. Int J Epidemiol 2001; 30:427-432.

13. Park S, Jee SH, Shin HR, Park EH, Shin A, Jung KW, et al. Attributable fraction of tobacco smoking on cancer using population-based nationwide cancer incidence and mortality data in Korea. BMC Cancer 2014;14:406.

14. Korean Supreme Court Decision, April 10, 2014. 2011Da22092 (Gong 2014Sang, 1004) [cited 2015 Mar 23]. Available from: http://library. scourt.go.kr/jsp/html/decision/16-oh,\%20kim\%202014.4.10.2011 Da22092.htm (Korean).

15. Broadbent A. Philosophy of epidemiology. New York: Palgrave Macmillan; 2013. p. 145-181.

16. Lee SG. Using epidemiological evidence to prove causation in toxic torts: recent cases of the Supreme Court of Korea. Justice 2015;146: 256-285 (Korean).

17. Hong YP, Kim SJ, Lew WJ, Lee EK, Han YC. The The seventh nationwide tuberculosis prevalence survey in Korea, 1995. Int J Tuberc Lung Dis 1998;2:27-36.

18. King AA, Ionides EL, Pascual M, Bouma MJ. Inapparent infections and cholera dynamics. Nature 2008;454:877-880.

19. Rothman KJ, Greenland S, Lash TL. Modern epidemiology. 3rd ed. Philadelphia: Lippincott Williams \& Wilkins; 2008. p. 5-31, 297.

20. Proctor RN. The history of the discovery of the cigarette-lung cancer link: evidentiary traditions, corporate denial, global toll. Tob Control 2012;21:87-91.

21. Wynder EL, Graham EA, Croninger AB. Experimental production of carcinoma with cigarette tar. Cancer Res 1953;13:855-864.

22. Brown \& Williamson. Report of progress-technical research department; 1952 [cited 2015 Apr 16]. Available from: http://legacy.library. ucsf.edu/tid/aql66b00.

23. Centers for Disease Control and Prevention. What are the risk factors for lung cancer? [cited 2015 Mar 23]. Available from: http://www. cdc.gov/cancer/lung/basic_info/risk_factors.htm.

24. Porta MS; International Epidemiological Association. A dictionary of epidemiology. 6th ed. New York: Oxford University Press; 2014. p. 135-136, 227.

25. Lee YG. Proving causation by epidemiologic data in toxic torts. Korean Lawyers Assoc J 2012;61:110-150 (Korean).

26. Greenland S. Relation of probability of causation to relative risk and doubling dose: a methodologic error that has become a social problem. Am J Public Health 1999;89:1166-1169.

27. Greenland S, Robins JM. Epidemiology, justice, and the probability of causation. Jurimetrics 2000;40:321-340.

28. Siemiatycki J, Karp I, Sylvestre MP, Pintos J. Estimating the proportion of cases of lung cancer legally attributable to smoking: a novel approach for class actions against the tobacco industry. Am J Public Health 2014;104:e60-e66.

29. Khang YH, Lynch JW, Kaplan GA. Health inequalities in Korea: ageand sex-specific educational differences in the 10 leading causes of death. Int J Epidemiol 2004;33:299-308.

30. Jung-Choi K, Khang YH, Cho HJ. Socioeconomic differentials in cause-specific mortality among 1.4 million South Korean public servants and their dependents. J Epidemiol Community Health 2011; 65:632-638.

31. Ministry of Health and Welfare, Korea Centers for Disease Control and Prevention. 2013 Korea health statistics. Sejong: Ministry of Health and Welfare; 2014, p. 74-77 (Korean).

32. Khang YH, Lynch JW, Jung-Choi K, Cho HJ. Explaining age-specific inequalities in mortality from all causes, cardiovascular disease and ischaemic heart disease among South Korean male public servants: relative and absolute perspectives. Heart 2008;94:75-82.

33. Moodie R, Stuckler D, Monteiro C, Sheron N, Neal B, Thamarangsi T, et al. Profits and pandemics: prevention of harmful effects of tobacco, alcohol, and ultra-processed food and drink industries. Lancet 2013;381:670-679.

34. Lim SS, Vos T, Flaxman AD, Danaei G, Shibuya K, Adair-Rohani H, et al. A comparative risk assessment of burden of disease and injury attributable to 67 risk factors and risk factor clusters in 21 regions, 1990-2010: a systematic analysis for the Global Burden of Disease Study 2010. Lancet 2012;380:2224-2260. 